\title{
How Plastic Mulch Types Affect Growth Parameters of Drip Irrigated Summer Peppers ${ }^{1}$
}

\author{
Megh R. Goyal, Carmen L. Santiago and Carmela Chao de Báez ${ }^{2}$ \\ ABSTRACT
}

Effects of plastic mulch types (transparent - T1, white - T2, black T3, silvercoated black plastic - T4), organic mulch (T5) and nonmulching (T6) were evaluated on growth parameters of drip irrigated summer peppers (Capsicum annuum L. cv. Cubanelle) in the semi-arid southern coast of Puerto Rico. Growth parameters (plant height, secondary and tertiary branches, number of nodes and buds on the primary branch) versus days after transplanting relationships were exponential. Transparent plastic caused lowest plant height and fruit weight. T2, T3, and T4 plots increased pepper yield by 28,46 and $132 \%$, respectively compared with non-mulched plots and accelerated flowering and fruit set. Relationships among plant height versus fresh pepper weight and growth parameters versus nutrient uptake were linear and the correlation coefficient varied from 0.90 to 0.99 .

\section{INTRODUCTION}

Plastic mulching may modify microclimate around the plant $(14,20$, $22,24)$ and may reduce weeds $(7,12,23)$ and insect-damaged fruits $(20)$. The use of plastic mulch has increased yield of strawberries $(23,24)$, squash (3), tomato (10), cucumber (10), and pepper (1,9). Goyal ${ }^{3}$ reported that silver coated black plastic mulch increased pepper yield compared to non-mulched plots during the winter. He suggested that plastic mulch modified humidity and soil temperature under the plant canopy. Jones et al. (14) indicated that plastic mulch reduced rainfall infiltration through the soil resulting in decreased nitrate losses and thus contributing to increased tomato yield. Chen and Katan (4) reported increases in soil temperature as soluble organic matter and nutrients in the plow zone with the use of transparent plastic. Dufault and Wiggans (8) found that white plastic caused early fruit-set, increases in pepper yield, and less vegetative growth than non-mulched plots. Anderson and Guttridge (1) observed that transparent and black plastics accelerated vegetative growth, plant height and fruit set of strawberries, due to moisture conservation. Gerald and Chambers (9) demonstrated increases in pepper yield with the use of reflective mulches.

Locasio et al. (16) indicated that the efficiency of nitrogen utilization increased from 8 to $42 \%$ with the use of plastic mulch. The $\mathrm{N}$ source,

\footnotetext{
${ }^{1}$ Manuscript submitted to Editorial Board October 7, 1983. This study was conducted under Southeast Regional Project S-143 (H326), "Trickle Irrigation in Humid RegionsPuerto Rico." Necessary help by Luis E. Rivera is duly acknowledged.

${ }^{2}$ Associate Agricultural Engineer, Research Assistant, and Associate Statistician, Agricultural Experiment Station, University of Puerto Rico, Mayagüez Campus, Río Piedras.

${ }^{3}$ M. R. Goyal, unpublished data.
} 
application rate and mulching increased pepper yield (18) compared with non-mulched plots. Similar results have been reported by Locasio and Fiskell (17). The use of black plastic advanced maturity, reduced incidence of diseases and increased the yield of many horticultural crops (2, $5,11,15,19,21)$. Clarkson $(5,6)$ found that $\mathrm{N}$ utilization was better in black plastic mulched corn. Geraldson (10) observed 20 to $30 \%$ increase in cucumber and tomato yield in plastic-mulched plots. Chaflant et al. (3) reported increases in summer squash yield with the use of plastic mulch. Johnson et al. (13) obtained increases in yield of pole beans under plastic mulch and drip irrigation. Goyal ${ }^{3}$ reported that plastic mulch increased winter pepper yield compared with summer peppers under drip irrigation.

The objectives of this study were to evaluate the effects of transparent, white, black, silver-coated black plastic, organic mulches and non-mulching on growth parameters of drip irrigated summer peppers in the semiarid region of Puerto Rico.

\section{MATERIALS AND METHODS}

This study was conducted at the Fortuna Agricultural Research and Development Center, Juana Diaz with drip irrigation systems described by Goyal elsewhere. Maximum and minimum air temperatures during the crop season were 33.4 and $15.0^{\circ} \mathrm{C}$. The precipitation and class $\mathrm{A}$ pan evaporation were 282.4 and $720.3 \mathrm{~mm}$, respectively. Pepper plants (var. Cubanelle, date of planting in nursery: February 12, 1982) were transplanted March 17, on both sides of a biwall drip line. Plant spacing was $30 \mathrm{~cm}$ down the row. Starting the 50th day, two plants per plot were removed each successive week to evaluate plant height, fresh and dry plant weight, fresh and dry fruit weight, number of nodes and buds on the primary, secondary and tertiary branches. The experiment was terminated July 20, 1982.

\section{RESULTS AND DISCUSSION}

In all treatments, relationships among pepper growth parameters (plant height, number of nodes, number of buds on primary branch, secondary and tertiary branches, dry plant weight) versus days after transplanting were exponential and followed a sigmoid growth curve as table 1 indicates. The curves exhibited the five growth phases, namely a lag phase (plants are being established), a log phase (growth rate is decreasing but plant still continues to grow), a point at which plant develops its maximum growth, and a senescence phase. Table 1 also indicates values of coefficient of determination $\left(\mathrm{R}^{2}\right)$ and regression coefficients. The regression coefficients were significant at the $5 \%$ level.

The differences among the treatments were nonsignificant at the $5 \%$ 
TABLE 1.-Effects of plastic mulch types on growth parameters in drip irrigated summer pepper $(1981-82)^{1}$

\begin{tabular}{|c|c|c|c|c|c|}
\hline \multirow{2}{*}{$\begin{array}{l}\text { Growth } \\
\text { Parameter }\end{array}$} & \multirow{2}{*}{ Treatment } & \multicolumn{3}{|c|}{ Regression coefficients ${ }^{2}$} & \multirow{2}{*}{$\mathrm{R}^{2}$} \\
\hline & & & $\mathrm{b}$ & c & \\
\hline \multirow[t]{6}{*}{ Plant height $(\mathrm{cm})$} & $\mathrm{T} 1$ & 65.45 & 5.68 & 0.037 & 0.82 \\
\hline & $\mathrm{T} 2$ & 74.96 & 7.23 & 0.039 & 0.90 \\
\hline & T3 & 75.48 & 5.77 & 0.036 & 0.85 \\
\hline & $\mathrm{T} 4$ & 76.51 & 7.79 & 0.045 & 0.91 \\
\hline & T5 & 83.24 & 6.54 & 0.036 & 0.84 \\
\hline & T6 & 74.29 & 7.58 & 0.039 & 0.87 \\
\hline \multirow{6}{*}{$\begin{array}{l}\text { No. of buds on the } \\
\text { primary branch }\end{array}$} & $\mathrm{T} 1$ & 32.80 & 4.60 & 0.061 & 0.78 \\
\hline & $\mathrm{T} 2$ & 33.90 & 5.75 & 0.082 & 0.79 \\
\hline & T3 & 34.68 & 5.07 & 0.077 & 0.78 \\
\hline & $\mathrm{T} 4$ & 34.89 & 5.77 & 0.085 & 0.83 \\
\hline & T5 & 33.33 & 7.04 & 0.096 & 0.76 \\
\hline & T6 & 33.52 & 5.55 & 0.080 & 0.80 \\
\hline \multirow{6}{*}{$\begin{array}{l}\text { Dry total weight } \\
\quad(\mathrm{g})\end{array}$} & $\mathrm{T} 1$ & 72.82 & 158.59 & 0.073 & 0.77 \\
\hline & $\mathrm{T} 2$ & 104.52 & 88.04 & 0.082 & 0.77 \\
\hline & T3 & 177.96 & 46.41 & 0.047 & 0.83 \\
\hline & $\mathrm{T} 4$ & 136.71 & 344.71 & 0.111 & 0.81 \\
\hline & T5 & 138.40 & 93.77 & 0.089 & 0.60 \\
\hline & T6 & 83.08 & 936.20 & 0.121 & 0.82 \\
\hline \multirow[t]{6}{*}{ No. of nodes } & $\mathrm{T} 1$ & 26.81 & 4.09 & 0.031 & 0.85 \\
\hline & $\mathrm{T} 2$ & 31.37 & 4.09 & 0.026 & 0.83 \\
\hline & T3 & 27.78 & 4.21 & 0.034 & 0.85 \\
\hline & $\mathrm{T} 4$ & 30.45 & 4.08 & 0.029 & 0.83 \\
\hline & T5 & 31.82 & 4.30 & 0.025 & 0.78 \\
\hline & $\mathrm{T} 6$ & 28.54 & 4.32 & 0.030 & 0.80 \\
\hline \multirow{6}{*}{$\begin{array}{l}\text { Secondary } \\
\text { branches }\end{array}$} & $\mathrm{T} 1$ & 23.0 & 43.7 & 0.044 & 0.78 \\
\hline & $\mathrm{T} 2^{3}$ & 7.44 & -0.103 & 0.002 & 0.74 \\
\hline & $\mathrm{T} 3$ & 27.4 & 18.4 & 0.034 & 0.74 \\
\hline & $\mathrm{T} 4$ & 102.9 & 31.5 & 0.019 & 0.79 \\
\hline & $\mathrm{T} 5^{3}$ & 7.9 & -0.14 & 0.002 & 0.77 \\
\hline & T6 & 21.0 & 35.9 & 0.049 & 0.83 \\
\hline \multirow[t]{6}{*}{ Tertiary branches } & $\mathrm{T} 1$ & 42.9 & 449.3 & 0.071 & 0.71 \\
\hline & $\mathrm{T} 2$ & 83.2 & 85.3 & 0.045 & 0.78 \\
\hline & T3 & 107.5 & 165.9 & 0.047 & 0.74 \\
\hline & $\mathrm{T} 4$ & 111.9 & 75.5 & 0.041 & 0.81 \\
\hline & $\mathrm{T} 5^{2}$ & 47.4 & -1.54 & 0.016 & 0.77 \\
\hline & $\mathrm{T} 6$ & 58.2 & 259.7 & 0.063 & 0.71 \\
\hline
\end{tabular}

${ }^{1} y=a /(1+b \exp (-c x)) ; x=$ days after transplanting; $y=$ growth parameter

${ }^{2}$ All regression coefficients were significant at the $5 \%$ probability level.

${ }^{3} y=a+b x+c x^{2}$

level in case of plant height on 9,44,50 and 64th day; number of nodes on $2,9,29,37,50,64,71,79,92,99$ and 106th day; number of buds on 9 , $16,29,44,50,64,71,92$ and 106th day; secondary branches on 71, 86, 92 and 106th day; tertiary branches on 50 and 92nd day; fresh plant weight 
TABLE 2.-Statistical differences among different plastic mulch types for various growth parameters in drip irrigated summer peppers

\begin{tabular}{|c|c|c|c|c|c|c|c|c|c|}
\hline \multirow{2}{*}{ Day } & \multirow{2}{*}{$\begin{array}{l}\text { Plant } \\
\text { height }\end{array}$} & \multirow{2}{*}{ Nodes } & \multirow{2}{*}{ Buds } & \multicolumn{2}{|c|}{ Branches } & \multicolumn{2}{|c|}{ Plant Weight } & \multicolumn{2}{|c|}{ Fruit Weight } \\
\hline & & & & Secondary & Tertiary & Fresh & Dry & Fresh & Dry \\
\hline 2 & $2<3$ & N.S. ${ }^{1}$ & $\begin{array}{l}5,6<1 \\
6<3\end{array}$ & $\mathrm{ND}^{1}$ & ND & $2<3,5,6$ & N.S. & ND & ND \\
\hline 9 & N.S. & N.S. & N.S. & ND & ND & N.S. & N.S. & ND & ND \\
\hline 16 & $2<4$ & $1,5<4$ & N.S. & ND & $\mathrm{ND}$ & $1,2,3,5,<4$ & $1,2,3,5,<4$ & ND & ND \\
\hline 22 & $2,6,<3$ & 14 & $1<4$ & ND & ND & $1<3$ & $1<3$ & ND & ND \\
\hline 29 & $5<3$ & N.S. & N.S. & ND & ND & $1<2,5$ & $1<5$ & ND & ND \\
\hline 37 & $\begin{array}{l}1,2<5 \\
6<3,5\end{array}$ & N.S. & $1<5$ & $1<2,3,4,5$ & $\begin{array}{l}14,5 \\
2,3,6,5\end{array}$ & $\begin{array}{l}1<4,5 \\
2,3,4,6<5\end{array}$ & $\begin{array}{l}1<4,5 \\
2,3,6<5\end{array}$ & ND & ND \\
\hline 44 & N.S. & $5<2,4$ & N.S. & $\begin{array}{c}1<2,3,4,5 \\
\quad 6<2\end{array}$ & 15 & $1<5$ & $1<5$ & $1<2,4,5$ & $1<2,4$ \\
\hline 50 & N.S. & N.S. & N.S. & $\begin{array}{c}1,3,6<4 \\
6<2\end{array}$ & N.S. & $\begin{array}{r}1<4,5 \\
6<4\end{array}$ & $1,6<4,5$ & N.S. & $\begin{array}{l}1<4 \\
6<4\end{array}$ \\
\hline 57 & $1<4$ & $1<4$ & $1<2,4,5$ & $\begin{array}{c}1<2,3,4,5,6 \\
3,6<5\end{array}$ & $\begin{array}{l}1,2,3,4,5 \\
62,3,4,5\end{array}$ & $\begin{array}{l}1<2,3,4,5 \\
6<4,5\end{array}$ & $\begin{array}{l}1<2,3,4,5 \\
6<4,5\end{array}$ & $\begin{array}{r}1<4,5 \\
6<4\end{array}$ & $1<3,4,5$ \\
\hline 64 & N.S. & N.S. & N.S. & $\begin{array}{l}1<2,3,4,5 \\
\quad 6<3\end{array}$ & $\begin{array}{c}1,2,3,4,5 \\
6<5\end{array}$ & $1<4,5$ & $\begin{array}{l}1,4,5 \\
2,3,6<5\end{array}$ & $1<4,5$ & $1<5$ \\
\hline 71 & $1<4$ & N.S. & N.S. & N.S. & $\begin{array}{r}12,4,5 \\
35,6\end{array}$ & $\begin{array}{l}1<2,4,5 \\
2,3,6<4 \\
3,6<5\end{array}$ & $\begin{array}{c}1,3<4,5 \\
6<4\end{array}$ & $\begin{array}{l}1<2,4,5 \\
2,3,5,6<4\end{array}$ & $\begin{array}{l}1,3<4,5 \\
6<4\end{array}$ \\
\hline 79 & $1,2,3,5,6<4$ & N.S. & $1,5,6<4$ & $1<4$ & $\begin{array}{r}14,5 \\
34\end{array}$ & $1,6<4$ & $1,3,6<4$ & $1,6<4$ & $1<4$ \\
\hline 86 & $\begin{array}{l}1,5<4 \\
5<2,3\end{array}$ & $5<3,6$ & $5<3,6$ & N.S. & $1,2,5,64$ & $\mathrm{DA}^{1}$ & $\mathrm{DA}$ & DA & DA \\
\hline 92 & $3<4$ & N.S. & N.S. & N.S. & N.S. & DA & DA & $\mathrm{DA}$ & DA \\
\hline 99 & $1<3,4,5,6$ & N.S. & $1,<3,4,6$ & $1<4$ & $1,4,5$ & DA & DA & DA & DA \\
\hline 106 & $1<5,3,4$ & N.S. & N.S. & N.S. & 15 & DA & DA & DA & DA \\
\hline 113 & $1<4,5$ & $1,6<4$ & $\begin{array}{l}1< \\
6<2,4\end{array}$ & $\begin{array}{l}1<2,4,5 \\
6<2,5\end{array}$ & $\begin{array}{l}1,6,4,5 \\
2,35\end{array}$ & DA & DA & $\mathrm{DA}$ & $\mathrm{DA}$ \\
\hline
\end{tabular}

${ }^{1} \mathrm{NS}=$ Not significant; $\mathrm{DA}=$ Data not analyzed; ND $=$ Data not taken.

Numbers in each column refer to treatment T1, T2, T3, T4, T5 and T6 respectively. Treatments were significant at $5 \%$ level as indicated above. 
on 9th day; dry plant weight on 2, 9th day; and fresh fruit weight on 50th day, respectively as indicated in table 2 . The differences among the treatments on other days were significant at $5 \%$ as table 2 shows.

Figures 1 and 2 show typical growth curves for plant height versus days after transplanting and number of buds versus days after transplant-

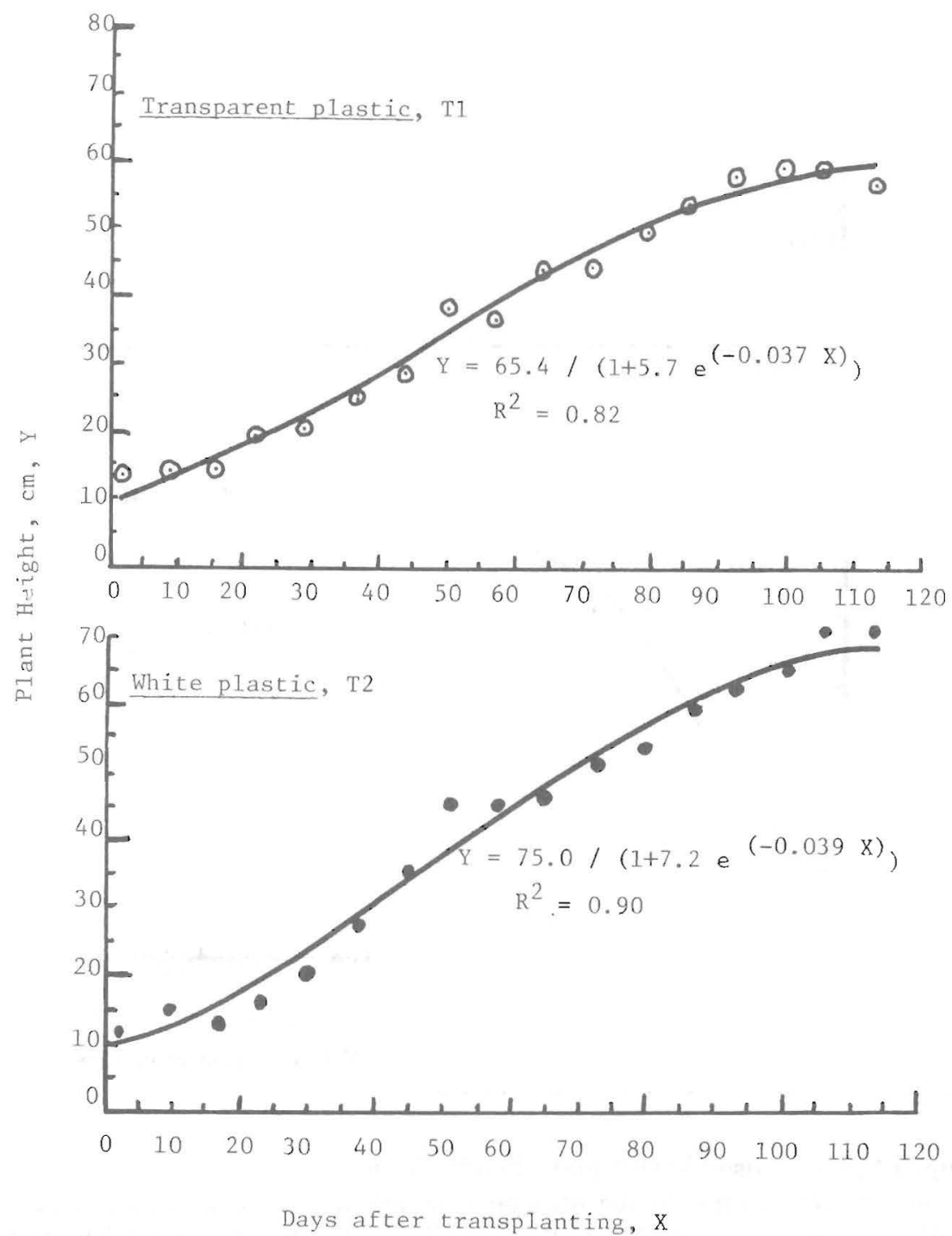

FIG. 1.-Effects of transparent and white plastic mulches on plant height of drip irrigated summer peppers. 


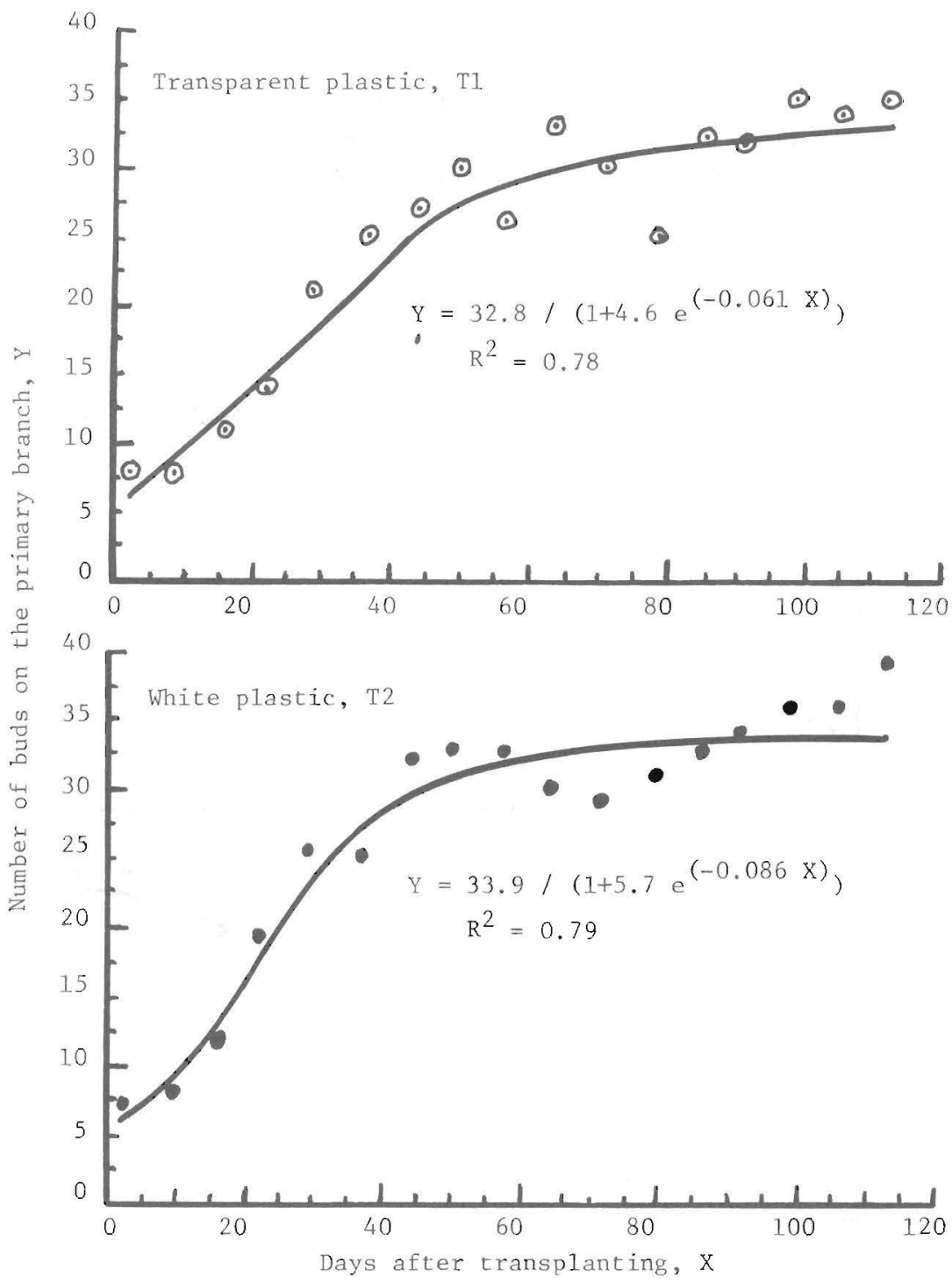

FIG. 2.-Effects of transparent and white plastic mulches on number of buds on the primary branch of drip irrigated summer peppers.

ing. T1 plots caused lowest plant height. This was attributed to too many weeds, which competed for nutrients, space and light, and higher daily soil temperatures at $0,7.5,15.0$ and $22.5 \mathrm{~cm}$ depth compared with other plots during the crop season. Number of buds increased at a decreasing rate after 40-45 days. This coincides with fruit-set during this period. 
TABLE 3.-Relationships among growth parameters and nutrient uptake in drip irrigated summer peppers under different plastic mulch types

\begin{tabular}{|c|c|c|c|c|c|}
\hline \multicolumn{2}{|c|}{ Variables $^{1}$} & \multirow{2}{*}{ Treatment } & \multicolumn{2}{|c|}{ Regression coefficients ${ }^{2}$} & \multirow{2}{*}{$\begin{array}{l}\text { Coefficient of } \\
\text { correlation, } r\end{array}$} \\
\hline$x$ & $\mathrm{y}$ & & & & \\
\hline \multirow[t]{6}{*}{ Plant height $(\mathrm{cm})$} & Fresh plant & $\mathrm{T} 1^{3}$ & -402.97 & 20.17 & 0.91 \\
\hline & weight (g) & $\mathrm{T} 2$ & -550.91 & 31.35 & 0.96 \\
\hline & & T3 & -690.78 & 33.33 & 0.95 \\
\hline & & $\mathrm{T} 4$ & -789.70 & 39.30 & 0.92 \\
\hline & & T5 & -686.80 & 36.10 & 0.93 \\
\hline & & T6 & -435.12 & 23.90 & 0.96 \\
\hline \multirow[t]{6}{*}{ Plant height $(\mathrm{cm})$} & Fresh fruit & $\mathrm{T} 1$ & -263.60 & 8.97 & 0.84 \\
\hline & weight (g/ & $\mathrm{T} 2$ & -514.63 & 15.92 & 0.94 \\
\hline & plant) & T3 & -547.96 & 15.97 & 0.91 \\
\hline & & $\mathrm{T} 4$ & -939.16 & 4.84 & 0.90 \\
\hline & & T5 & -543.47 & 15.76 & 0.91 \\
\hline & & T6 & -401.07 & 11.99 & 0.95 \\
\hline \multirow{2}{*}{$\begin{array}{l}\text { Fresh plant } \\
\text { weight (g) }\end{array}$} & N uptake (g/ & $\mathrm{T} 2$ & +0.14 & 0.002 & 0.99 \\
\hline & plant) & T3 & -0.014 & 0.0025 & 0.99 \\
\hline \multirow{2}{*}{$\begin{array}{l}\text { Fresh plant } \\
\text { weight }(\mathrm{g})\end{array}$} & K uptake (g/ & $\mathrm{T} 2$ & +0.21 & 0.003 & 0.97 \\
\hline & plant) & T3 & +0.04 & 0.0034 & 0.99 \\
\hline \multirow{2}{*}{$\begin{array}{l}\text { Fresh plant } \\
\text { weight (g) }\end{array}$} & Ca uptake $(\mathrm{g} /$ & $\mathrm{T} 2$ & +0.02 & 0.001 & 0.99 \\
\hline & plant) & $\mathrm{T} 3$ & -0.009 & 0.0012 & 0.97 \\
\hline \multirow{2}{*}{$\begin{array}{l}\text { Fresh plant } \\
\text { weight }(\mathrm{g})\end{array}$} & Mg uptake (g/ & $\mathrm{T} 2$ & +0.004 & 0.0003 & 0.99 \\
\hline & plant) & T3 & +0.0118 & 0.00034 & 0.97 \\
\hline \multirow{2}{*}{$\begin{array}{l}\text { Fresh plant } \\
\text { weight }(\mathrm{g})\end{array}$} & P uptake (g/ & $\mathrm{T} 2$ & -0.01 & 0.0004 & 0.91 \\
\hline & plant) & $\mathrm{T} 3$ & -0.0053 & 0.00044 & 0.98 \\
\hline
\end{tabular}

${ }^{1} y=a+b x$.

${ }^{2}$ All regression coefficients were significant at the $5 \%$ probability level.

${ }^{3} \mathrm{~T} 1$ = Transparent plastic; $\mathrm{T} 2=$ white plastic; $\mathrm{T} 3=$ Black plastic; $\mathrm{T} 4=$ Silver-coated black plastic; T5 = Organic mulch; T6 $=$ Control.

Table 3 shows the linear relationships among pepper growth parameters versus nutrient uptake in different plots and indicates values of regression coefficients and coefficient of correlation. High correlation coefficient among fresh fruit weight versus plant height provides a nondestructive method to estimate pepper yield.

Our results agree with Dufautt and Wiggans (8) who found increases in pepper yield and observed early flowering with the use of plastic mulch. In our study, pepper flowering was observed on the 29th day in the T2, T3 and T4 plots followed by T5, T6 plots.

\section{RESUMEN}

El efecto de diferentes tipos de cubiertas (plástica transparente-T1, plástica blanca-T2, plástica negra-T3, plástica plateada-T4 y orgánica T5 y sin cubierta-T6) se evaluó en los parámetros de crecimiento de pimientos (Capsicum annuum cv Cubanelle) regados por goteo durante el verano. El experimento se hizo en Juana Díaz, en la costa semiárida del sur de Puerto 
Rico. Los parámetros de crecimiento (altura de las plantas, ramas secundarias y terciarias, número de yemas y nudos en las ramas terciarias mostraron una relación exponencial) en contraposición de dias después del trasplante. El plástico transparente causó los valores más bajos en la altura de las plantas y en el peso de las frutas. Los tratamientos T2, T3 y T4 incrementaron el rendimiento de pimientos en 28,46 , y $132 \%$, respectivamente, al compararlo con el tratamiento sin cubierta plástica; además, aceleraron la floración y el cuaje de las frutas. La relación entre la altura de las plantas y el peso fresco de los pimientos y los parámetros de crecimiento contra la absorción de nutrimentos fue lineal y el coeficiente de correlación varió de .90 a .99 .

\section{LITERATURE CITED}

1. Anderson, H. M. and C. G. Guttridge, 1978. The performance of strawberries on polyethylene-mulched ridges in England, Hort. Res., 18: 27-39.

2. Carolus, R. L., 1958. Raise your yield: use black plastic, Am. Veg. Grower, 6 (3): 16.

3. Chalfant, R. B., C. A. Jeworski, A. W. Johnson and D. R. Summer, 1977. Reflective film mulches, millet barriers, and pesticide effects on watermelon mosaic virus, insects, nematodes, soil-borne fungi, and yield of yellow summer squash, J. Am. Soc. Hort. Sci. 102: 11-5.

4. Chen, Y. and J. Katan, 1980. Effect of solar heating of soils by transparent polyethylene mulching on their chemical properties, Soil Sci., 130 (5): 271-76.

5. Clarkson, V. A., 1958. Plastic mulch for increased yields, Oregon Veg. Digest 7 (1): 4.

6. Clarkson, V. A., 1960. Effect of black polyethylene mulch on soil and microclimate temperature and nitrate level. Soil Sci. Am. J., 52 (6): 307-09.

7. Cox, J. E. and V. C. Austen, 1963. Experiments on weed control in strawberry beds, Agric. Gaz. N. S. W., 74: 90-94, 105.

8. Dufault, R. and J. C. Wiggans, 1981. Response of sweet peppers to solar reflectors and reflective mulches, Hort. Sci. 16 (1): $65-7$.

9. Gerald, C. J. and G. Chambers, 1967. Effect of reflective coatings on soil temperature, soil moisture, and the establishment of fall bell peppers, Agron. J. 59: 293-96.

10. Geraldson, C. M., 1962. Growing tomatoes and cucumbers with high analysis fertilizer and plastic mulch, Proc. Fla. State Hort. Sci. 75: 253-60.

11. Hamma, S. and F. J. McAxalle, 1958. Black plastic for white spear pear production, Am. Veg. Grower, 6 (5): 45.

12. Jowarski, C. A., S. C. Phatak, A. W. Johnson and S. M. McCarter, 1981. Tomato production and soil pest control in relation to width of fumigated and mulched bed and soil fumigation rate, Hort. Sci. 16 (5): 667-69.

13. Johnson, A. W., C. A. Jaworski, D. R. Summer and R. B. Chaflant, 1979. Effects of film mulch, trickle irrigation, and soil pesticides on nematodes and yield of polebeans, Plant Dis. Rep 63: 360-64.

14. Jones, T. L., U. S. Jones and D. O. Ezell, 1977. Effect of nitrogen and plastic mulch on properties of troup loamy sand and on yield of "water" tomatoes, J. Am. Soc. Hort. Sci. 102 (3): 273-75.

15. Kelley, J., 1959. Growing asters by using a black mulch, Florist Review, CXXIV (No. 3202: 61).

16. Locasio, S. J., J. G. A. Fiskell, D. A. Graetz and R. O. Hauck, 1977. Nitrogen uptake by pepper as influenced by mulch and time of fertilizer application. Agron. Abstr. 161. 
17. —and-, 1979. Pepper response to sulfur-coated urea, mulch and nitrogen rate, Proc. Fla. State Hort. Soc. 20: 112-15.

18. — - and F. G. Martin, 1981. Responses of bell pepper to nitrogen sources, J. Am. Hort. Sci. 106 (5): 628-32.

19. Mack, H. J., 1958. Black plastic increases bush bean yields, Oregon Veg. Dig. 7 (4): 5.

20. Porter, L. A., 1962. Polyethylene sheet as mulch for strawberries, N. Z. FL. Agric. 105: $247-55$.

21. Sheldrake, S., 1958. Black plastic mulch, Cornell Veg. News, 8 (2): 3.

22. Thompson, B. D., 1960. Response of strawberries to mulching with plastic, Proc. Fl. State Hort. Soc. 72: 179-85.

23. Voth, V. and R. S. Bringhurst, 1959. Polyethylene over strawberries, Calif. Agric. 13 (5): $5-14$.

24. - and — 1961. Pruning and polyethylene mulching of summer planted strawberries in southern California, Proc. Am. Soc. Hort. Sci. 78: 275-80. 